Musées, Patrimoine et Culture scientifiques et techniques

\title{
Wikipédia ou le paradoxe du muséologue
}

\section{François Mairesse}

URL : http://journals.openedition.org/ocim/1738

DOI : 10.4000/ocim. 1738

ISSN : 2108-646X

\section{Éditeur}

OCIM

Édition imprimée

Date de publication : 1 janvier 2017

Pagination : 33-35

ISSN : 0994-1908

Référence électronique

François Mairesse, «Wikipédia ou le paradoxe du muséologue », La Lettre de l'OCIM [En ligne],

169 | 2017, mis en ligne le 01 janvier 2018, consulté le 19 avril 2019. URL : http://

journals.openedition.org/ocim/1738; DOI : 10.4000/ocim.1738

Ce document a été généré automatiquement le 19 avril 2019.

Tous droits réservés 


\title{
Wikipédia ou le paradoxe du muséologue
}

\author{
François Mairesse
}

1 L'encyclopédie en ligne Wikipédia est devenue une référence obligée du Web, tant pour le grand public que pour les chercheurs qui l'utilisent régulièrement (sans pour autant s'y référer, au vu de la volatilité de son contenu). La version française, riche de plus d'un million d'articles, comporte un grand nombre de contributions sur des personnalités plus ou moins médiatiques (de Nabilla Benattia à Homer Simpson, en passant bien sûr par Louis Pasteur ou Jean-Paul II). Si tout le monde a déjà consulté ce site, plus rares sont les lecteurs qui disposent d'une réelle connaissance sur son fonctionnement particulier, et notamment sur le rôle ou l'identité de ses contributeurs, son statut collaboratif ainsi que sur ses règles d'édition - collaboration ne signifiant pas ipso facto participation libre de toute contrainte.

2 L'ouvrage Wikipédia. Objet scientifique non identifié arrive à point nommé, car il existe encore peu de références sur le fonctionnement de Wikipédia dont la logique a totalement bousculé le monde des encyclopédies traditionnelles comme en témoigne l'arrêt des éditions papier de la Britannica et de l'Universalis en 2012. La respectabilité et le sérieux de l'entreprise, longtemps décriée par les milieux académiques, ont été progressivement reconnus à la fin de la première décennie du millénaire, grâce notamment au soutien de Chris Anderson, auteur du best seller La longue traîne, analysant le modèle sous-jacent au travail collaboratif de dizaines de milliers d'internautes, et la diminution des risques d'erreurs en résultant ${ }^{1}$. De fait, comme le souligne Alexandre Moatti dans un chapitre consacré aux postures d'opposition à l'encyclopédie (p. 123-133), les mouvements de contestation, en France, se développent essentiellement entre 2007 et 2009, pour diminuer à partir de 2010.

3 L'ouvrage Wikipédia. Objet scientifique non identifié rassemble ainsi les contributions d'une douzaine de chercheurs, essentiellement français, dans le cadre d'un projet de recherche entamé en 2012 sur l'évaluation de la fameuse encyclopédie en ligne. Le caractère très récent du phénomène (en regard de la Britannica, éditée à partir du XVIIIe siècle) ne permet encore qu'une première appréciation, et sa croissance exponentielle ne 
saurait pour autant présager du futur de l'entreprise, dont l'essoufflement est déjà évoqué (p. 109). Si les premiers articles se proposent d'identifier le phénomène et d'en décrire les contours, le corps de l'ouvrage (les deuxième et troisième parties) porte essentiellement sur le fonctionnement de l'encyclopédie en ligne, soit la gouvernance de Wikipédia, ainsi que sur les questions d'autorité et de légitimité qui lui sont liées. La dernière partie, quant à elle, aborde l'encyclopédie comme terrain d'observation, soit pour y rassembler des informations et en vérifier la fiabilité, soit pour analyser d'un point de vue ethnographique les rapports entre les contributeurs.

4 La partie qui intéressera sans doute le plus les professionnels de musées, pour peu qu'ils cherchent à ce que leur établissement (ou leurs propres réalisations) soit référencé dans l'encyclopédie, porte sur les règles qui prévalent à la rédaction des articles. Car si tout un chacun est chaleureusement invité à apporter sa pierre à l'édifice (en créant ou en modifiant un article), le travail wikipédien repose sur un système de contraintes spécifiques à chaque catégorie de sujets, qu'il s'agisse d'une notice portant sur un responsable politique, une organisation ou un auteur, ces contraintes visant à éviter que l'encyclopédie ne soit utilisée comme un outil d'autopromotion, la neutralité de point de vue en étant l'un des cinq principes fondateurs et directeurs. Parallèlement, un système de gouvernance assez sophistiqué a été mis en place, privilégiant la discussion et le consensus à l'autorité et se fondant tout à la fois sur l'existence d'une communauté structurée et l'expérience d'administrateurs expérimentés. Le corpus des règles relatives au choix des articles, leur rédaction, l'utilisation des sources, les citations, la bonne conduite - et les sanctions, pour ceux qui les enfreignent - a été mis en place afin de faciliter les discussions autour de la pertinence d'un article. L'objectif du système vise à régler les conflits inévitables liés à la rédaction d'articles sur des sujets sensibles - mais il peut arriver que des querelles homériques aient eu lieu à partir de sujets apparemment anodins ${ }^{2}$. Si le système permet de résoudre la plupart des conflits sans avoir besoin de recourir à l'intervention d'une autorité hiérarchique, il produit néanmoins un certain nombre de conséquences qui ne sont pas sans susciter notre interrogation. D'une part, l'existence d'un tel corpus réglementaire suppose qu'il soit assimilé et connu, ce que les rédacteurs les plus aguerris ont tourné à leur avantage pour influer sur l'écriture d'autres articles : "Une caste de wikipédiens s'est progressivement consolidée en exerçant un pouvoir d'intimidation par la règle (wikilawyering), que renforce notamment la loi des appariements sélectifs qui contribue à renforcer la solidarité entre ceux qui ont un fort niveau d'édition", remarque Dominique Cardon (p. 31). D'autre part, l'approche épistémologique privilégiée par Wikipédia repose sur la vérifiabilité plutôt que sur la vérité. Cette approche, se fondant sur la neutralité et l'objectivité, apparaît pour certains (Jankowsky, cité par Langlais p. 78) obsolète et liée à un encyclopédisme positiviste remontant au XIXe siècle. Ainsi, Antonio Casilli évoque dans son article sa déconvenue lorsque, annonçant la mort de son collègue Claude Lévi-Strauss, il voit sa contribution refusée : "L'argument d'autorité, le fait d'écrire de l'intérieur de la même institution dans laquelle Claude Lévi-Strauss avait enseigné, ne semblait pas recevable. La page fut modifiée quelques temps après par une personne pouvant inclure un lien avec la dépêche AFP annonçant la mort du chercheur" (p. 94).

Un tel système de règles apparaît sans doute inévitable ; il permet en tout état de cause de comprendre pourquoi il semble plus aisé de publier un article sur Nabilla Benattia que sur un conservateur ou un muséologue de renom, ce qui semble somme toute assez paradoxal pour une encyclopédie se définissant comme "universelle". Les critères de notoriété en la 
matière sont simples : pour qu'un article portant sur une personnalité puisse être retenu, cette dernière doit "avoir été l'objet principal d'un ouvrage publié à compte d'éditeur ou d'au moins deux articles ou émissions, espacés d'au moins deux ans et diffusés dans des médias d'envergure nationale ou internationale" (ce qui est bien le cas de Nabilla...) ou être mentionnée dans une encyclopédie de référence. L'existence de ces sources secondaires dites "de qualité" constitue l'une des conditions sine qua non à toute admissibilité. Si un tel système de validation s'avère assez simple et cohérent pour une personnalité médiatique, il apparaît en revanche plus difficile à mettre en œuvre pour des personnalités à la renommée plus discrète ou plus confidentielle, comme peuvent l'être celles des musées et de la muséologie. Ainsi, à titre d'exemple, un article proposé sur Michel Van-Praët a mis plus de cinq années à obtenir l'approbation de l'équipe en charge de veiller à la mise en ligne des nouveaux articles.

6 À plusieurs égards, Michel Van-Praët peut être considéré comme une personnalité reconnue dans le monde des sciences et de la muséologie, tant pour ses qualités académiques que muséales. Ayant intégré en 1974 le Muséum national d'Histoire naturelle en qualité d'enseignant-chercheur, il rédige une quarantaine de publications sur les cnidaires dont il devient l'un des spécialistes. Conjuguant à la fois le point de vue des scientifiques à travers sa profession de biologiste et celui des publics en qualité de directeur du service d'Action pédagogique et culturelle du Muséum, c'est surtout pour sa participation à la rénovation de la galerie de Zoologie qu'il est reconnu par la communauté muséale. Il rédige ainsi dans le courant de l'été 1986 le synopsis de la Galerie de l'Évolution, inaugurée en 1994. Il assure également de 1996 à 2002 la coordination muséologique du projet de rénovation des galeries d'Anatomie comparée et de Paléontologie du Muséum au terme duquel il est nommé directeur du Département des galeries. Après un passage par l'Inspection générale des musées de France, il dirige de 2010 à 2012 l'équipe de rénovation du musée de l'Homme en vue d'en relancer le programme. Président du Bureau du Comité national français de l'ICOM de 1999 à 2004, il devient membre de la Commission internationale de déontologie de l'ICOM entre 2000 et 2010 et participe à ce titre à la rédaction des deux dernières versions du code de déontologie. Il est également élu au conseil exécutif de l'ICOM où il effectue deux mandats successifs, de 2004 à 2007 et de 2007 à 2010. Par ailleurs, il crée et co-dirige avec Jean Davallon entre 1993 et 1995 le premier diplôme français de 3e cycle de muséologie, le Diplôme d'études approfondies (DEA) "Publics, Expositions, Musées" entre l'université Jean Monnet de Saint-Étienne, le Conservatoire national des arts et métiers (CNAM) et le Muséum national d'Histoire naturelle. Puis, en 1995, il participe à la création du DEA de "Muséologie : sciences et sociétés" au Muséum national d'Histoire naturelle avant d'en assurer la direction de 1999 à 2004.

Jusqu'à une date très récente, ce parcours scientifique n'a cependant pas mérité les honneurs de Wikipédia, la communauté des internautes ayant rejeté dès 2011 un premier article rédigé sur le muséologue initiateur de la rénovation de la Grande Galerie, aucune des références secondaires citées par les rédacteurs n'ayant été jugées suffisamment notables. Il existe en effet une procédure spécifique permettant aux contributeurs de voter pour ou contre la suppression d'une page, procédure durant laquelle cinq avis défavorables ont été formulés, ayant pour résultat final la clôture de la page. Il est intéressant de relever les commentaires des internautes, pour le moins édifiants ${ }^{3}$. Bien qu'un connaisseur de l'œuvre de Michel Van-Praët ait fourni un certain nombre de références (17 passages évoquant les activités du scientifique sur Internet) ainsi qu'une 
liste d'environ 160 articles scientifiques ou de vulgarisation établissant la notoriété du muséologue, les commentaires évoquent "un professeur de muséologie qui a très peu publié", "une carrière essentiellement administrative" ainsi qu'une "notoriété très faible, manifestement pas un universitaire de référence dans son domaine". D'autres supposent une potentielle "autopromotion". Ite missa est. Le projet renaît toutefois en janvier 2016, un nouvel article étant proposé par un contributeur. Mais, une fois encore, la proposition se voit refusée par les Wikipédiens, au motif qu'aucun argument nouveau ne permet d'envisager son éventuelle admission. L'affaire en restera là pendant plusieurs mois... jusqu'à ce qu'en décembre de la même année, la communauté wikipédienne relance le débat en vue de l'admissibilité de l'article. Les échanges, parfois virulents, débouchent cette fois sur la remise d'une quinzaine d'avis, dont neuf se révèlent favorables à la publication de l'article, officiellement mis en ligne le 15 décembre, suivant une structure et des références en grande partie identiques à celles proposées en début d'année.

Cette anecdote, qui témoigne de l'évolution des jugements subjectifs au sein de la communauté wikipédienne plutôt que de la notoriété objective des personnalités représentées (qui, dans le cas présent, n'a guère évolué), illustre sans doute certaines des limites de l'encyclopédie en ligne ; elle permet surtout de comprendre pourquoi certaines personnalités, bien qu'importantes dans leur secteur, n'y sont pas (encore) présentes. Peu d'internautes s'interrogent réellement sur le fonctionnement de cette entreprise encyclopédique comportant des centaines de milliers de pages numériques sur les sujets les plus divers, ainsi que sur le travail de ses principaux contributeurs. Ceux-ci ont à faire face quotidiennement à des dizaines de propositions d'articles, dont un grand nombre relèvent davantage d'une logique d'autopromotion (notamment de la part d'entreprises ou de responsables politiques) que du respect des règles établies par Diderot et d'Alembert dans l'Encyclopédie ou Dictionnaire raisonné des sciences, des arts et des métiers. Il n'en reste pas moins vrai que les règles édictées pour garantir le sérieux de l'entreprise sont appliquées parfois de manière paradoxale.

9 Dans un tel contexte, Wikipédia. Objet scientifique non identifié présente le mérite de chercher à explorer et comprendre le fonctionnement, l'intérêt et les limites de ce site Internet, devenu en quelques années une référence incontournable en matière de connaissances.

\section{NOTES}

1. Anderson, C. La longue traîne. Paris : Pearson, 2009.

2. Voir l'article Wikipédia : guerres d'édition les plus futiles sur l'encyclopédie en ligne.

3. Voir le fil de dialogue dans les archives des pages à supprimer <https://fr.wikipedia.org/wiki/ Discussion:Michel_Van_Pra\%C3\%ABt/Suppression> 


\section{RÉSUMÉS}

À propos de l'ouvrage Wikipédia. Objet scientifique non identifié de Lionel Barbe, Louise Merzeau et Valérie Schaffer (Paris : Presses universitaires de Paris Ouest, 2015, 214 p.)

INDEX

Mots-clés : wikipédia

\section{AUTEUR}

\section{FRANÇOIS MAIRESSE}

professeur des universités, Sorbonne Nouvelle-Paris 3 et Karine Robé-Ramette, conférencière et spécialiste en archéologie orientale 